\title{
ON FREE SUBALGEBRAS OF CERTAIN DIVISION ALGEBRAS
}

\author{
MARTIN LORENZ
}

\begin{abstract}
Using ideas of Makar-Limanov, we construct noncommutative free subalgebras in certain division algebras which arise as fields of fractions of skew polynomial rings. Our result applies in particular to the Weyi division algebra $D_{1}$.
\end{abstract}

The goal of this note is to prove the following

THEOREM. Let $K=k(t)$ be the rational function field over the field $k$, let $\alpha \in$ Aut $_{\ell}(K)$ be of infinite order, and let $K_{\alpha}[X]$ denote the skew polynomial ring. Then the classical division ring of fractions $D=Q\left(K_{\alpha}[X]\right)$ contains noncommutative free k-subalgebras.

For example, if $\alpha$ sends $t$ to $\lambda \cdot t$, where $\lambda \in k^{*}$ is not a root of unity, then $D=Q\left(K_{\alpha}[X]\right)$ equals the division algebra $E_{\lambda}$ studied in [L] and the theorem gives [L, Theorem 2.2]. Also, if $t^{\alpha}=t-1$ and char $k=0$, then $D=Q\left(K_{\alpha}[X]\right)$ is the Weyl division algebra $D_{1}=Q(k[X, Y] /(X Y-Y X-1))($ take $t=X Y)$. Thus we also recover the main result of Makar-Limanov's article [ML1]. We emphasize, however, that the crucial ideas used in the proof of the theorem come directly from Makar-Limanov's papers [ML1 and ML2]. In fact, if $k$ is algebraically closed, then every $\alpha \in$ Aut $_{\mathscr{X}}(K)$ of infinite order is conjugate to one of the above two types of automorphisms and so the theorem could be deduced from the above special cases. Thus we only take credit for the presentation and the unified approach to MakarLimanov's results.

Throughout, $k$ will denote a commutative field.

1. Lemmas on $\operatorname{Aut}_{k}(k(t))$. Let $K=k(t)$ be the rational function field over $k$. Then every $\sigma \in$ Aut $_{\ell}(K)$ acts by a fractional linear transformation

$$
t \mapsto \frac{a t+b}{c t+d} \quad \text { with } \quad\left(\begin{array}{ll}
a & b \\
c & d
\end{array}\right)=: M(\sigma ; t) \in \operatorname{PGL}_{2}(k)
$$

The effect of replacing the primitive element $t$ of $K / k$ by $t^{\tau}, \tau \in$ Aut $_{k}(K)$, is described by the formula

$$
M\left(\sigma ; t^{\tau}\right)=M(\tau ; t) \cdot M(\sigma ; t) \cdot M(\tau ; t)^{-1}
$$

Received by the editors August 20, 1985 and, in revised form, October 14, 1985.

1980 Mathematics Subject Classification (1985 Revision). Primary 16A39, 16A05; Secondary 16A06, 16A27, 17B35. 
$\mathrm{PGL}_{2}(k)$ also operates by fractional linear transformations on $\mathbf{P}^{1}(k)=k \cup\{\infty\}$, and $\infty$ is a fixed point for $\left(\begin{array}{cc}a & b \\ c & d\end{array}\right) \in \mathrm{PGL}_{2}(k)$ if and only if $c=0$.

Lemma 1. Let $K=\ell(t)$ and let $\sigma \in$ Aut $_{\ell}(K)$ be such that $S=M(\sigma ; t)$ satisfies $S^{i} \cdot \infty \neq \infty$ for all $i \neq 0$. Then $\left\{f^{\sigma}-f \mid f \in K\right\} \cap k[t] \subseteq k$.

Proof. Let $f \in K$ be given with $f^{\sigma}-f \in k[t]$ and let $A$ be the set of poles in $\mathbf{P}^{1}(k)$ for the function $f^{\sigma}$. Then clearly $B=S \cdot A$ is the set of poles for $f$ and any element in the symmetric difference $D=(A \cup B) \backslash(A \cap B)$ is a pole for $f^{\sigma}-f$. But $|A|=|B|$ so $|D|=2(|A|-|A \cap B|)$ is an even integer. By assumption, $f^{\sigma}-f$ has at most one pole, so $|D|$ being even must be zero. Hence $A=A \cap B=B$. This says that $S$ stabilizes the finite set $A$ and hence some power of $S$ fixes all points of $A$. Thus, by assumption, $\infty \notin A=B$. But then $\infty$ is not a pole of the polynomial $f^{\sigma}-f$ so $f^{\sigma}-f$ must be a constant, which proves the lemma.

Lemma 2. Let $K=k(t)$ and let $\sigma \in \mathrm{Aut}_{\ell}(K)$ be of infinite order. Then there exists a primitive element a for $K / \hbar$ such that $A=M(\sigma ; a)$ satisfies $A^{i} \cdot \infty \neq \infty$ for all $i \neq 0$.

Proof. Set $T=M(\sigma ; t) \in \mathrm{PGL}_{2}(k)$. It suffices to show that there exists $\xi \in$ $\mathbf{P}^{1}(k)=k \cup\{\infty\}$ with $T^{i} \cdot \xi \neq \xi$ for all $i \neq 0$. Indeed, if $\xi$ is such an element, choose $X \in \mathrm{PGL}_{2}(k)$ with $X \cdot \xi=\infty$ and set $a=t^{\tau}$, where $M(\tau ; t)=X$. Then $a$ is a primitive element for $K / k$, and $A=M(\sigma ; a)=X T X^{-1}$ satisfies $A^{i} \cdot \infty \neq \infty$ for all $i \neq 0$, as required.

As is well known, if $T \in \mathrm{PGL}_{2}(k)$ has three fixed points in $\mathbf{P}^{1}(k)$ then it must be the identity. Now look at the union of the fixed points of all $T^{i}$ with $i \neq 0$. If there are at least three points in this union then, say, $T^{a}$ fixes $u, T^{b}$ fixes $v$, and $T^{c}$ fixes $w$. Then $T^{a b c}$ fixes $u, v, w$ so $T^{a b c}=1$ and $T$ has finite order, contradiction. Since $\left|\mathbf{P}^{1}(k)\right| \geqslant 3$, there must be a point not fixed by any power of $T$. This proves the lemma.

ExAmples. (a) If $\sigma$ is given by $t^{\sigma}=t+\mu, \mu \in k^{*}$ and char $k=0$, then $T=$ $M(\sigma ; t)$ satisfies $T^{i} \cdot \xi \neq \xi$ for all $\xi \in \ell$ and $i \neq 0$. Thus we can take $X=\left(\begin{array}{cc}0 & 1 \\ 1 & -\xi\end{array}\right)$ in the above argument and get $a=(t-\xi)^{-1}(\xi \in k)$ as possible choices for $a$.

(b) Similarly, if $t^{\sigma}=\lambda t, \lambda \in k^{*}$ a nonroot of unity, then we can take $a$ of the form $\alpha=(t-\xi)^{-1}\left(\xi \in k^{*}\right)$.

2. Proof of the theorem. Let $K=h(t)$ and let $\alpha \in$ Aut $_{k}(K)$ be of infinite order. Set $D=Q\left(K_{\alpha}[X]\right)$, the classical division algebra of fractions of the skew polynomial ring $K_{\alpha}[X]$. The theorem will be a consequence of the following

Proposition. Let $a \in K$ be as in Lemma 2 and set $b=a(1-X)^{-1} \in D$. Then the subalgebra $k[a, b] \subseteq D$ is free on $\{a, b\}$.

The rest of this section is devoted to the proof of this proposition. We will proceed in a number of steps. First note that the localization of the skew power series ring $K_{\alpha} \llbracket x \rrbracket$ at the powers of $X$ is a skew field containing $K_{\alpha}[X]$, and hence a copy of $D$. Here, $(1-X)^{-1} \in D$ corresponds to $1+X+X^{2}+\cdots \in K_{\alpha} \llbracket X \rrbracket$ and so the images of $a$ and $b$ lie in $K_{\alpha} \llbracket X \rrbracket$. In the following, we work in $K_{\alpha} \llbracket X \rrbracket$. Note that, as 
$K$-vector space, $K_{\alpha} \llbracket X \rrbracket$ can be identified with the set of functions $\varphi: \mathbf{N}_{0}=$ $\{0,1,2, \ldots\} \rightarrow K$.

(A) Equivalence Classes of Functions $\mathbf{N}_{0} \rightarrow K$. Let $\mathscr{F}$ denote the set of equivalence classes of functions $\varphi: \mathbf{N}_{0} \rightarrow K$ modulo the relation $\sim$ given by

$$
\varphi \sim \psi: \Leftrightarrow \varphi(n)=\psi(n) \text { for almost all } n .
$$

Then $\mathscr{F}$ becomes a commutative ring under pointwise addition and multiplication of functions. In the sequel, we use the same notation for a function $\varphi: \mathbf{N}_{0} \rightarrow K$ and its class modulo $\sim$. For $\varphi \in \mathscr{F}$ define $\varphi^{\sigma} \in \mathscr{F}$ by

$$
\varphi^{\sigma}(n)=\varphi(n+1) \quad(n \gg 0) .
$$

Then $\sigma$ is an automorphism of $\mathscr{F}$ which acts trivially on the set of constant functions $\mathscr{C} \subseteq \mathscr{F}$. Clearly, $\mathscr{C} \cong K$ and we will identify constants with their values in $K$. Finally, define $\gamma \in \mathscr{F}$ by

$$
\gamma(n)=a^{\alpha^{n}} \quad(n \gg 0),
$$

and let $\mathscr{A} \subseteq \mathscr{F}$ be the subring of $\mathscr{F}$ generated by $\mathscr{C}$ and $\gamma$.

ClaIM. $\mathscr{A}=\mathscr{C}[\gamma]$ is a polynomial algebra over $\mathscr{C}$. Moreover, all nonzero elements of $\mathscr{A}$ are units in $\mathscr{F}$ and so $\mathscr{K}:=Q(\mathscr{A}) \subseteq \mathscr{F}$. Finally, $\sigma$ operates on $\mathscr{K}$ and $\left\{\varphi^{\sigma}-\varphi \mid \varphi \in \mathscr{X}\right\} \cap \mathscr{A} \subseteq \mathscr{C}$.

PROOF. First note that $\gamma: \mathbf{N}_{0} \rightarrow K$ is injective, because otherwise $a$ would have a finite $\alpha$-orbit and hence $\alpha$ would have finite order. Therefore, each nontrivial polynomial $\sum_{i \in I} c_{i} \gamma^{i}\left(I \neq \varnothing, c_{i} \in \mathscr{C}^{*}\right)$ has only finitely many zeros in $\mathbf{N}_{0}$. It follows that $\sum_{i \in I} c_{i} \gamma^{i}$ is a unit in $\mathscr{F}$. In particular, $\gamma$ is transcendental over $\mathscr{C}$ and $\mathscr{X}=Q(\mathscr{A}) \cong \mathscr{C}(\gamma) \subseteq \mathscr{F}$. Moreover, if $M(\alpha ; a)=\left(\begin{array}{ll}r & s \\ u & v\end{array}\right) \in \mathrm{PGL}_{2}(k)$, then for all $n \gg 0$ we have

$$
\gamma^{\sigma}(n)=\left(a^{\alpha}\right)^{\alpha^{n}}=\left(\frac{r a+s}{u a+v}\right)^{\alpha^{n}}=\frac{r \gamma(n)+s}{u \gamma(n)+v}
$$

so that $\gamma^{\sigma}=(r \gamma+s) /(u \gamma+v) \in \mathscr{K}=\mathscr{C}(\gamma)$ and $M(\sigma ; \gamma)=M(\alpha ; a)$. Therefore, by our choice of $a$, we have $M(\sigma ; \gamma)^{i} \cdot \infty \neq \infty$ for all $i \neq 0$ and Lemma 1 implies that $\left\{\varphi^{\alpha}-\varphi \mid \varphi \in \mathscr{X}\right\} \cap \mathscr{C}[\gamma] \subseteq \mathscr{C}$.

(B) For $J=\left(j_{1}, j_{2}, \ldots, j_{s}\right) \in \mathbf{N}^{s}(s \geqslant 1)$ set

$$
\beta_{J}(n)=\sum_{n \geqslant n_{1} \geqslant \cdots \geqslant n_{s} \geqslant 0} \gamma\left(n_{1}\right)^{j_{1}} \cdot \gamma\left(n_{2}\right)^{j_{2}} \cdots \gamma\left(n_{s}\right)^{j_{s}} \quad\left(n \in \mathbf{N}_{0}\right) .
$$

For $s \leqslant 0$ let $J=(\varnothing)$ and $\beta_{(\varnothing)}(n)=1$ for all $n$.

Claim. In order to prove the proposition, it suffices to show that the functions $\beta_{J} \in \mathscr{F}\left(J \in \bigcup_{s} \mathbf{N}^{s}\right)$ are linearly independent over $\mathscr{K} \subseteq \mathscr{F}$.

Proof. We have to show that formally distinct monomials in $a$ and $b=$ $a(1-X)^{-1}=a\left(1+X+X^{2}+\cdots\right)$ are linearly independent over $k$. Each such monomial has the form

$$
m_{\left(i_{0}, i_{1}, \ldots, i_{v}\right)}=a^{i_{0}}(1-X)^{-1} a^{i_{1}} \cdots(1-X)^{-1} a^{i_{v}},
$$


where $0 \leqslant v=\# b$-factors, $i_{v} \in \mathbf{N}_{0}$ and $i_{0}, \ldots, i_{v-1} \in \mathbf{N}$ for $v \geqslant 1$. In $K_{\alpha} \llbracket X \rrbracket$, we have

$$
\begin{aligned}
m_{\left(i_{0}, i_{1}, \ldots, i_{n}\right)} & =\sum_{n \geqslant 0} X^{n}\left(a^{\alpha^{n}}\right)^{i_{0}} \cdot\left(\sum_{n \geqslant n_{1} \geqslant \cdots \geqslant n^{\prime-1} \geqslant 0}\left(a^{\alpha^{n_{1}}}\right)^{i_{1}} \cdots\left(a^{\alpha^{n-1}}\right)^{i_{v^{\prime}-1}}\right) a^{i_{n}} \\
& =\sum_{n \geqslant 0} X^{n} \gamma(n)^{i_{0}} \cdot a^{i_{v}} \cdot \beta_{\left(i_{1}, \ldots, i_{v-1}\right)}(n)
\end{aligned}
$$

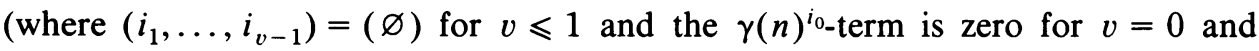
$n>0)$. For each multi-index $I=\left(i_{0}, i_{1}, \ldots, i_{v}\right)$ as above write $I^{\prime}=\left(i_{1}, \ldots, i_{v-1}\right) \in$ $\mathbf{N}^{v-1}\left(I^{\prime}=(\varnothing)\right.$ for $\left.v \leqslant 1\right)$. Suppose that $\sum_{I \in \mathscr{I}} \xi_{I} m_{I}\left(\xi_{I} \in k\right)$ is a $k$-linear relation between different monomials $m_{I}(I \in \mathscr{I})$ and set $\mathscr{I}_{0}=\left\{\left(i_{0}, \ldots, i_{v}\right) \in \mathscr{I} \mid v=0\right\}$, $\mathscr{I}_{>0}=\mathscr{I} \backslash \mathscr{I}_{0}$. Then, in $\mathscr{F}$, we have $m_{I}=0$ for all $I \in \mathscr{I}_{0}$ and $\Sigma_{I \in \mathscr{I}>0} \xi_{i} \gamma^{i_{0}} a^{i{ }} \beta_{I^{\prime}}=$ 0 . For $I \in \mathscr{I}_{>0}, \xi_{I} \gamma^{i_{0}} a^{i}$ belongs to $\mathscr{K}$ and $\xi_{I} a^{i_{v}} \in \mathscr{C}$. By assumption, the $\beta_{I^{\prime}}$ 's are $\mathscr{K}$-independent. Furthermore, by step (A), $\gamma$ is transcendental over $\mathscr{C}$. Finally, $a$ is transcendental over $k$, by definition. Thus we conclude that $\xi_{I}=0$ for all $I \in \mathscr{I}_{>0}$ and so the given relation reduces to $\sum_{I \in \mathscr{I}_{0}} \xi_{I} m_{I}=0$. But this is a polynomial equation for $a$ over $k$ which must be trivial. Therefore, $\xi_{I}=0$ for all $I \in \mathscr{I}_{0}$ also, as it was to be shown.

(C) Conclusion. For $\varphi \in \mathscr{F}$ define $\Delta \varphi \in \mathscr{F}$ by $\Delta \varphi=\varphi^{\sigma}-\varphi$. The following formulas are straightforward to verify:

$$
\begin{array}{cc}
\Delta(\varphi \psi)=\varphi^{\sigma} \cdot \Delta \psi+\Delta \varphi \cdot \psi & (\varphi, \psi \in \mathscr{F}), \\
\Delta \beta_{J}=\sum_{l=1}^{s}\left(\gamma^{j_{1}+\cdots+j_{l}}\right)^{\sigma} \cdot \beta_{J(l)} & (=0 \text { for } s=0),
\end{array}
$$

where $J=\left(j_{1}, \ldots, j_{k}\right) \in \mathbf{N}^{s}$ and $J(l):=\left(j_{l+1}, \ldots, j_{s}\right)$.

Moreover, by step (A), we have $\Delta \mathscr{K} \cap \mathscr{C}[\gamma] \subseteq \mathscr{C}$. Now suppose that $\Sigma_{J} e_{J} \beta_{J}=0$ $\left(J \in \cup_{s \geqslant 0} \mathbf{N}^{s}, e_{J} \in \mathscr{K}\right.$ almost all $=0$ ) is a nontrivial $\mathscr{K}$-linear relation which is chosen so that $u=\max \left\{l(J) \mid e_{J} \neq 0\right\}$ is minimal, where we have set $l(J)=s$ for $J \in \mathbf{N}^{s}(s \geqslant 0)$, and the number of summands with $l(J)=u$ is minimal. Clearly, $u \geqslant 1$. Rewrite the above relation by isolating the "long" $J$ 's to the left:

$$
\sum_{J \in \mathscr{J}} e_{J} \beta_{J}=\sum_{J \in U_{s<u} \mathrm{~N}^{s}} d_{J} \beta_{J}
$$

where $\varnothing \neq \mathscr{J} \subseteq \mathbf{N}^{u}$ and all $e_{J}(J \in \mathscr{J})$ are nonzero. We can and will also assume that $e_{J}=1$ for some $J \in \mathscr{J}$. Using (i) and (ii) above, we see that

(iii) $\Delta\left(e_{J} \beta_{J}\right)=\Delta e_{J} \cdot \beta_{J}+\delta$, where $\Delta e_{J} \in \mathscr{K}$ and $\delta$ is a $\mathscr{K}$-linear combination of $\beta_{I}$ 's with $l(I)<l(J)$.

As $e_{J}=1$ for some $J \in \mathscr{J}$, and hence $\Delta e_{J}=0$, the relation

$$
\Delta\left(\sum_{J \in \mathscr{J}} e_{J} \beta_{J}\right)=\Delta\left(\sum_{J \in \cup_{s<u} \mathbf{N}^{s}} d_{J} \beta_{J}\right)
$$

involves fewer summands with $l(J)=u$. Therefore, our minimality assumption implies that the latter relation is trivial. In particular, in view of (iii), we deduce that $\Delta e_{J}=0$ for all $J \in \mathscr{J}$, i.e., $e_{J} \in \mathscr{C}^{*}$ for all $J \in \mathscr{J}$. Moreover, for all $J=$ $\left(j_{1}, j_{2}, \ldots, j_{u}\right) \in \mathscr{J}$, the coefficients of $\beta_{J(1)}$ (recall that $\left.J(1)=\left(j_{2}, \ldots, j_{u}\right)\right)$ in (iv) 
must be equal on both sides, i.e.

$$
\Delta d_{J(1)}=\sum_{\substack{I=\left(i_{1}, \ldots, i_{u}\right) \in \mathscr{J} \\ I(1)=J(1)}} e_{I}^{\sigma}\left(\gamma^{i_{1}}\right)^{\sigma}
$$

holds for all $J \in \mathscr{J}$. Thus, as $e_{I} \in \mathscr{C}$ for all $I$ on the right, we see that

$$
\left(\Delta d_{J(1)}\right)^{\sigma^{-1}}=\left(d_{J(1)}^{\sigma^{-1}}\right)=\sum_{\substack{I=\left(i_{1}, \ldots, i_{u}\right) \in \mathscr{J} \\ I(1)=J(1)}} e_{I} \gamma^{i_{1}}
$$

belongs to $\Delta \mathscr{K} \cap \gamma \mathscr{C}[\gamma]=0$. But, by step (A), $\gamma$ is transcendental over $\mathscr{C}$ and so all $e_{I}$ in (v) must be 0 , contradiction! This completes the proof of the proposition, and hence the theorem is proved.

ACKNOWLEDGMENT. Research supported by the DeutscheForschungsgemeinschaft/Heisenberg Programm (Lo 261/2-1). The author is indebted to the referee for the present simple proofs of Lemmas 1 and 2.

\section{REFERENCES}

[ML1] L. Makar-Limanov, The skew field $D_{1}$ contains free subalgebras, Comm. Algebra 11 (1983), 2003-2006.

[ML2] __, On group rings of nilpotent groups, preprint.

[L] M. Lorenz, Group rings and division rings, Methods in Ring Theory (F. van Oystayen, ed.), Reidel, Dordrecht, 1984, pp. 265-280.

Max-Planck-Institut für Mathematik, Gottfried-Claren-Strasse 26, D-5300 Bonn 3, Federal REPUBLIC OF GERMANY 